\title{
Catch Me if You Can: Evaluating Android Anti-malware against Transformation Attacks
}

\author{
Vaibhav Rastogi*, Yan Chen, and Xuxian Jiang
}

\begin{abstract}
Mobile malware threats (e.g., on Android) have recently become a real concern. In this paper, we evaluate the state-of-the-art commercial mobile anti-malware products for Android and test how resistant they are against various common obfuscation techniques (even with known malware). Such an evaluation is important for not only measuring the available defense against mobile malware threats but also proposing effective, next-generation solutions. We developed DroidChameleon, a systematic framework with various transformation techniques, and used it for our study. Our results on ten popular commercial anti-malware applications for Android are worrisome: none of these tools is resistant against common malware transformation techniques. Moreover, a majority of them can be trivially defeated by applying slight transformation over known malware with little effort for malware authors. Finally, in the light of our results, we propose possible remedies for improving the current state of malware detection on mobile devices.
\end{abstract}

Index Terms-Mobile, malware, anti-malware, Android.

\section{INTRODUCTION}

Mobile computing devices such as smartphones and tablets are becoming increasingly popular. Unfortunately, this popularity attracts malware authors too. In reality, mobile malware has already become a serious concern. It has been reported that on Android, one of the most popular smartphone platforms [2], malware has constantly been on the rise and the platform is seen as "clearly today's target" [3], [4]. With the growth of malware, the platform has also seen an evolution of antimalware tools, with a range of free and paid offerings now available in the official Android app market, Google Play.

In this paper, we aim to evaluate the efficacy of anti-malware tools on Android in the face of various evasion techniques. For example, polymorphism is used to evade detection tools by transforming a malware in different forms ("morphs") but with the same code. Metamorphism is another common technique that can mutate code so that it no longer remains the same but still has the same behavior. For ease of presentation, we use the term polymorphism in this paper to represent both obfuscation techniques. In addition, we use the term 'transformation' broadly, to refer to various polymorphic or metamorphic changes.

Polymorphic attacks have long been a plague for traditional desktop and server systems. While there exist earlier studies

Copyright (c) 2013 IEEE. Personal use of this material is permitted. However, permission to use this material for any other purposes must be obtained from the IEEE by sending a request to pubs-permissions@ieee.org.

A conference version of this paper appears in ASIACCS'13 [1]

V. Rastogi and Y. Chen are with Northwestern University, e-mail: vrastogi@u.northwestern.edu,ychen@northwestern.edu

X. Jiang is with North Carolina State University, e-mail: jiang@cs.ncsu.edu

${ }^{*}$ Corresponding author on the effectiveness of anti-malware tools on PCs [5], our domain of study is different in that we exclusively focus on mobile devices like smartphones, which require different ways for anti-malware design. Also, malware on mobile devices have recently escalated their evolution but the capabilities of existing anti-malware tools are largely not yet understood. In the meantime, simple forms of polymorphic attacks have already been seen in the wild [6].

To evaluate existing anti-malware software, we develop a systematic framework called DroidChameleon with several common transformation techniques that may be used to transform Android applications automatically. Some of these transformations are highly specific to the Android platform only. Based on the framework, we pass known malware samples (from different families) through these transformations to generate new variants of malware, which are verified to possess the originals' malicious functionality. We use these variants to evaluate the effectiveness and robustness of popular anti-malware tools.

Our results on ten popular anti-malware products, some of which even claim resistance against malware transformations, show that all the anti-malware products used in our study have little protection against common transformation techniques. The techniques themselves are simple. The fact that even without much technical difficulty, we can evade anti-malware tools, highlights the seriousness of the problem. Many of them succumb to even trivial transformations such as repacking or reassembling that do not involve any code-level transformation. Our results also give insights about detection models used in existing anti-malware and their capabilities, thus shedding light on possible ways for their improvements. We hope that our findings work as a wake-up call and motivation for the community to improve the current state of mobile malware detection.

To summarize, this paper makes the following contributions.

- We systematically evaluate anti-malware products for Android regarding their resistance against various transformation techniques in known malware. For this purpose, we developed DroidChameleon, a systematic framework with various transformation techniques to facilitate anti-malware evaluation.

- We have implemented a prototype of DroidChameleon and used it to evaluate ten popular anti-malware products for Android. Our findings show that all of them are vulnerable to common evasion techniques. Moreover, we find that $90 \%$ of the signatures studied do not require static analysis of bytecode. 
- We studied the evolution of anti-malware tools over a period of one year. Our findings show that some antimalware tools have tried to strengthen their signatures with a trend towards content-based signatures while previously they were evaded by trivial transformations not involving code-level changes. The improved signatures are however still shown to be easily evaded.

- Based on our evaluation results, we also explore possible ways to improve current anti-malware solutions. Specifically, we point out that Android eases developing advanced detection techniques because much code is highlevel bytecodes rather than native codes. Furthermore, certain platform support can be enlisted to cope with advanced transformations.

The rest of this paper is organized as follows. We present in Section II the necessary background and detail in Section III the DroidChameleon design. We then provide implementation details in Section IV and summarize our malware and antimalware data sets in Section V. After that, we present our findings in Section VI, followed by a brief discussion in Section VII on how to improve current anti-malware solutions. Finally, we examine related work in Section VIII and conclude in Section IX.

\section{BACKGROUND}

Android is an operating system for mobile devices such as smartphones and tablets. It is based on the Linux kernel and provides a middleware implementing subsystems such as telephony, window management, management of communication with and between applications, managing application lifecycle, and so on.

Applications are programmed primarily in Java though the programmers are allowed to do native programming via JNI (Java native interface). Instead of running Java bytecode, Android runs Dalvik bytecode, which is produced from Java bytecode. In Dalvik, instead of having multiple . class files as in the case of Java, all the classes are packed together in a single . dex file.

Android applications are made of four types of components, namely activities, services, broadcast receivers, and content providers. These application components are implemented as classes in application code and are declared in the AndroidManifest (see next paragraph). The Android middleware interacts with the application through these components.

Android application packages are jar files containing the application bytecode as a classes. dex file, any native code libraries, application resources such as images, config files and so on, and a manifest, called AndroidManifest. It is a binary XML file, which declares the application package name, a string that is supposed to be unique to an application, and the different components in the application. It also declares other things (such as application permissions) which are not so relevant to the present work. The AndroidManifest is written in human readable XML and is transformed to binary XML during application build.

Only digitally signed applications may be installed on an Android device. Signing keys are usually owned by individual developers and not by a central authority, and there is no chain of trust. All third party applications run unprivileged on Android.

\section{FRAMEWORK DESIGN}

In this work, we focus on the evaluation of anti-malware products for Android. Specifically, we attempt to deduce the kind of signatures that these products use to detect malware and how resistant these signatures are against changes in the malware binaries. In this paper, we generally use the term transformation to denote semantics preserving changes to a program. Since we are dealing with malware, we only care about the interested semantics such as sending SMS message to a premium number and not things like change of application name in the system logs.

In this work, we develop several different kinds of transformations that may be applied to malware samples while preserving their malicious behavior. Each malware sample undergoes one or more transformations and then passes through the anti-malware tools. The detection results are then collected and used to make deductions about the detection strengths of these anti-malware tools.

We classify our transformations as trivial (which do not require code level changes), those which result in variants that can still be detected by static analysis (DSA), and those which can render malware undetectable by static analysis (NSA). In the rest of this section, we describe the different kinds of transformations that we have in the DroidChameleon framework. Where appropriate we give examples, using original and transformed code. Transformations for Dalvik bytecode are given in Smali (as in Listing 1), an intuitive assembly language for Dalvik bytecode.

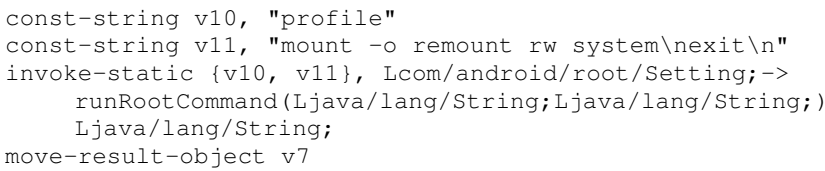

Listing 1: A code fragment from DroidDream malware

\section{A. Trivial Transformations}

Trivial transformations do not require code-level changes. We have the following transformations in this category.

1) Repacking: Recall that Android packages are signed jar files. These may be unzipped with the regular zip utilities and then repacked again with tools offered in the Android SDK. Once repacked, applications are signed with custom keys (the original developer keys are not available). Detection signatures that match the developer keys or a checksum of the entire application package are rendered ineffective by this transformation. Note that this transformation applies to Android applications only; there is no counterpart in general for Windows applications although the malware in the latter operating systems are known to use sophisticated packers for the purpose of evading anti-malware tools. 
2) Disassembling and Reassembling: The compiled Dalvik bytecode in classes.dex of the application package may be disassembled and then reassembled back again. The various items (classes, methods, strings, and so on) in a dex file may be arranged or represented in more than one way and thus a compiled program may be represented in different forms. Signatures that match the whole classes.dex are beaten by this transformation. Signatures that depend on the order of different items in the dex file will also likely break with this transformation. Similar assembling/disassembling also applies to the resources in an Android package and to the conversion of AndroidManifest between binary and human readable formats.

3) Changing Package Name: Every application is identified by a package name unique to the application. This name is defined in the package's AndroidManifest. We change the package name in a given malicious application to another name. Package names of apps are concepts unique to Android and hence similar transformations do not exist in other systems.

\section{B. Transformation Attacks Detectable by Static Analysis (DSA)}

The application of DSA transformations does not break all types of static analysis. Specifically, forms of analysis that describe the semantics, such as data flows are still possible. Only simpler checks such as string matching or matching API calls may be thwarted.

1) Identifier Renaming: Most class, method, and field identifiers in bytecode can be renamed. We note that several free obfuscation tools such as ProGuard [7] provide identifier renaming. Listing 2 presents an example transformation for code in Listing 1.

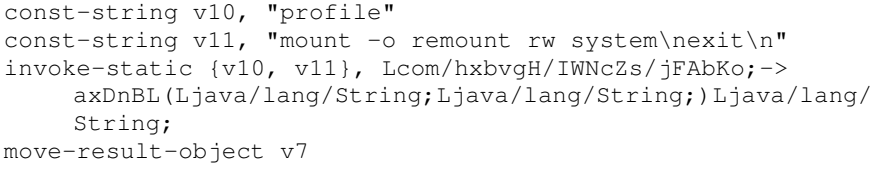

Listing 2: Code in Listing 1 after identifier renaming

2) Data Encoding: The dex files contain all the strings and array data that have been used in the code. These strings and arrays may be used to develop signatures against malware. To beat such signatures we can keep these in encoded form. Listing 3 shows code in Listing 1, transformed by string encoding.

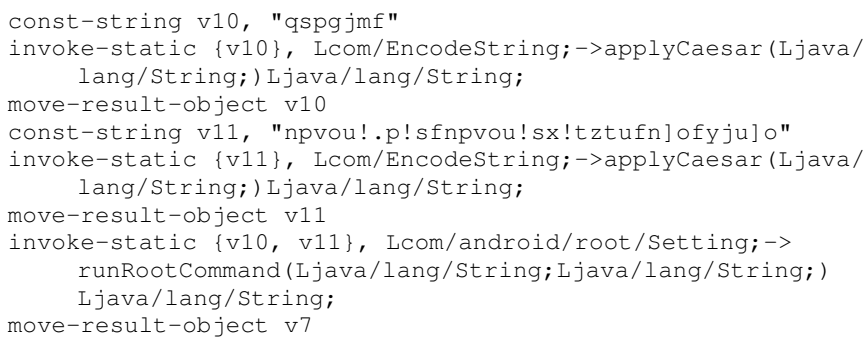

Listing 3: Code in Listing 1 after string encoding. Strings are encoded with a Caesar cipher of shift +1 .
3) Call Indirections: This transformation can be seen as a simple way to manipulate call graph of the application to defeat automatic matching. Given a method call, the call is converted to a call to a previously non-existing method that then calls the method in the original call. This can be done for all calls, those going out into framework libraries as well as those within the application code. This transformation may be seen as trivial function outlining [8].

4) Code Reordering: Code reordering reorders the instructions in the methods of a program. This transformation is accomplished by reordering the instructions and inserting goto instructions to preserve the runtime execution sequence of the instructions. Listing 4 shows an example reordering.

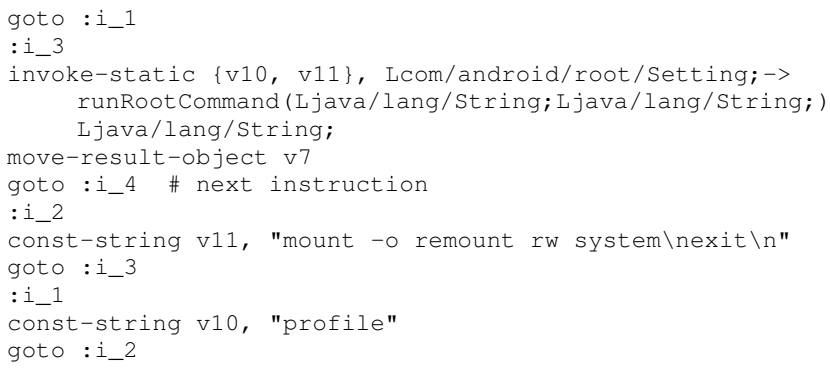

Listing 4: Code in Listing 1 reverse ordered

5) Junk Code Insertion: These transformations introduce code sequences that are executed but do not affect rest of the program. Detection based on analyzing instruction (or opcode) sequences may be defeated by junk code insertion. Junk code may constitute simple nop sequences or more sophisticated sequences and branches that actually have no effect on the semantics.

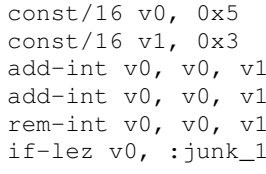

Listing 5: An example of a junk code fragment

6) Encrypting Payloads and Native Exploits: In Android, native code is usually made available as libraries accessed via JNI. However, some malware such as DroidDream also pack native code exploits meant to run from a command line in non-standard locations in the application package. All such files may be stored encrypted in the application package and be decrypted at runtime. Certain malware such as DroidDream also carry payload applications that are installed once the system has been compromised. These payloads may also be stored encrypted. We categorize payload and exploit encryption as DSA because signature based static detection is still possible based on the main application's bytecode. These are easily implemented and have been seen in practice as well (e.g., DroidKungFu malware uses encrypted exploit).

7) Other Simple Transformations: There are a few other transformations as well, specific to Android. Debug information, such as source file names, local and parameter variable names, and source line numbers may be stripped off. Moreover, non-code files and resources contained in Android packages may be renamed or modified. 
8) Composite Transformations: Any of the above transformations may be combined with one another to generate stronger obfuscations. While compositions are not commutative, anti-malware detection results should be agnostic to the order of application of transformations in all cases discussed here.

\section{Transformation Attacks Non-Detectable by Static Analysis (NSA)}

These transformations can break all kinds of static analysis. Some encoding or encryption is typically required so that no static analysis scheme can infer parts of the code. Parts of the encryption keys may even be fetched remotely. In this scenario, interpreting or emulating the code (i.e., dynamic analysis) is still possible but static analysis becomes infeasible.

1) Reflection: The Java reflection API allows a program to invoke a method by using the name of the methods. We can convert any method call into a call to that method via reflection. This makes it difficult to analyze statically which method is being called. A subsequent encryption of the method name can make it impossible for any static analysis to recover the call.

Listing 6 illustrates code in Listing 1 after reflection transformation.

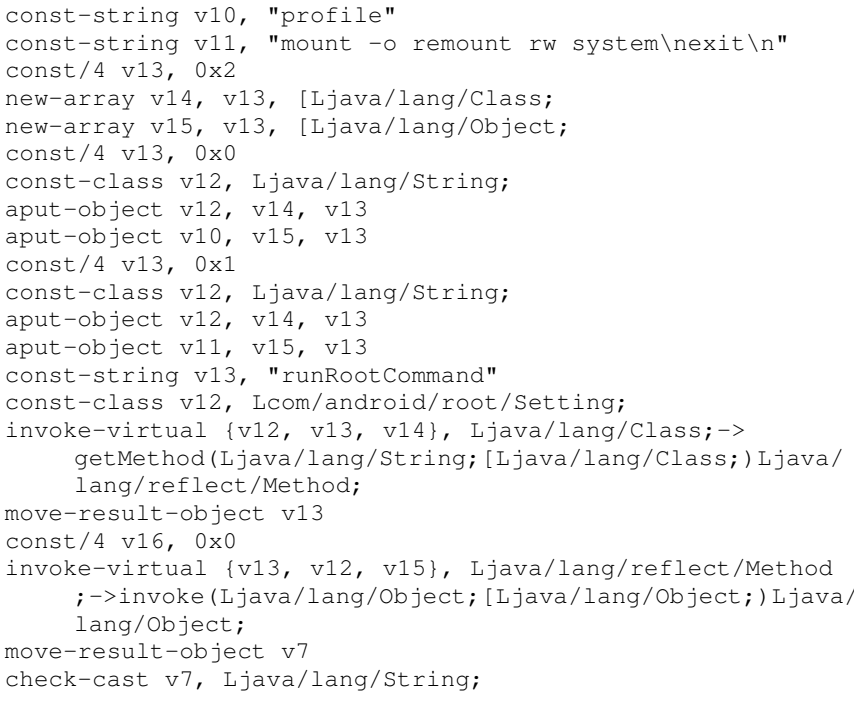

Listing 6: Listing 1 with method call by reflection

2) Bytecode Encryption: Code encryption tries to make the code unavailable for static analysis. The relevant piece of the application code is stored in an encrypted form and is decrypted at runtime via a decryption routine. Code encryption has long been used in polymorphic viruses; the only code available to signature based antivirus applications remains the decryption routine, which is typically obfuscated in different ways at each replication of the virus to evade detection. We discuss here code encryption alone; obfuscation of the decryption routine may be possible by other methods discussed above.

We accomplish bytecode encryption by moving most of the application in a separate dex file (packed as a jar) and storing it in the application package in an encrypted form. When one of the application components (such as an activity or a service) is created, it first calls a decryption routine that decrypts the dex file and loads it via a user defined class loader. In Android, the DexClassloader provides the functionality to load arbitrary dex files. Following this operation, calls can be made into the code in the newly loaded dex file. Alternatively, one could define a custom class loader that loads classes from a custom file format, possibly containing encrypted classes. We note that classes which have been defined as components need to be available in classes. dex (one that is loaded by default) so that they are available to the Android middleware in the default class loader. These classes then act as wrappers for component classes that have been moved to other dex files.

\section{IMPLEMENTATION}

We applied all the above DroidChameleon transformations to the malware samples. We have implemented most of the transformations so that they may be applied automatically to the application. Automation implies that the malware authors can generate polymorphic malware at a very fast pace. Certain transformations such as native code encryption are not possible to completely automate because one needs to know how native code files are being handled in the code. ${ }^{1}$ Transformations that require modification of the AndroidManifest (rename packages and renaming components) have not been completely automated because we felt it was more convenient to modify manually the AndroidManifest for our study. Nevertheless, it is certainly possible to automate this as well. Finally, we did not automate bytecode encryption, although there are no technical barriers to doing that. However, we have implemented a proofof-concept bytecode encryption transformation manually on existing malware.

We utilize the Smali/Baksmali [9] and its companion tool Apktool [10] for our implementation. Our code-level transformations are implemented over Smali. Moreover, disassembling and assembling transformation uses Apktool. This has the effect of repacking, changing the order and representation of items in the classes.dex file, and changing the AndroidManifest (while preserving the semantics of it). All other transformations in our implementation (apart from repacking) make use of Apktool to unpack/repack application packages. Our overall implementation comprises about 1,100 lines of Python and Scala code.

We verified that our implementation of transformations do not modify the semantics of the programs. Specifically, we tested our transformations against several test cases and verified their correctness on two malware samples, DroidDream and Fakeplayer. In general, verifying correctness on actual malware is challenging because some of the original samples have turned non-functional owing to, for example, the remote server not responding, and because being able to detect all the malicious functionality requires a custom, appropriately monitored environment. Indeed, our original DroidDream sample would not work because it failed to get a reply from a remote

\footnotetext{
${ }^{1}$ Native code stored in non standard locations is typically copied from the application package to the application directory by the application itself (possibly through an available Android API).
} 
TABLE I: Anti-malware products evaluated.

\begin{tabular}{lllll}
\hline Vendor & Product & Package name & Version & \# downloads \\
AVG & Antivirus Free & com.antivirus & 3.1 & $50 \mathrm{M}-100 \mathrm{M}$ \\
Symantec & Norton Mobile Security & com.symantec.mobilesecurity & 3.3 .0 .892 & $5 \mathrm{M}-10 \mathrm{M}$ \\
Lookout & Lookout Mobile Security & com.lookout & 8.7 .1 -EDC6DFS & $10 \mathrm{M}-50 \mathrm{M}$ \\
ESET & ESET Mobile Security & com.eset.ems & 1.1 .995 .1221 & $500 \mathrm{~K}-1 \mathrm{M}$ \\
Dr. Web & Dr. Web anti-virus Light & com.drweb & 7.00 .3 & $10 \mathrm{M}-50 \mathrm{M}$ \\
Kaspersky & Kaspersky Mobile Security & com.kms & 9.36 .28 & $1 \mathrm{M}-5 \mathrm{M}$ \\
Trend micro & Mobile Security Personal Ed. & com.trendmicro.tmmspersonal & 2.6 .2 & $100 \mathrm{~K}-500 \mathrm{~K}$ \\
ESTSoft & ALYac Android & com.estsoft.alyac & 1.3 .5 .2 & $5 \mathrm{M}-10 \mathrm{M}$ \\
Zoner & Zoner Antivirus Free & com.zoner.android.antivirus & 1.7 .2 & $1 \mathrm{M}-5 \mathrm{M}$ \\
Webroot & Webroot Security \& Antivirus & com.webroot.security & 3.1 .0 .4547 & $500 \mathrm{~K}-1 \mathrm{M}$ \\
\hline
\end{tabular}

TABLE II: Malware samples used for testing anti-malware tools

\begin{tabular}{|c|c|c|c|c|}
\hline Family & Package name & SHA-1 code & Date found & Remarks \\
\hline DroidDream & $\begin{array}{l}\text { com.droiddream. bowl- } \\
\text { ingtime }\end{array}$ & $\begin{array}{l}72 \mathrm{adcf} 43 \mathrm{e} 5 \mathrm{f} 945 \mathrm{ca} 9 \mathrm{f} 72 \\
064 \mathrm{~b} 81 \mathrm{dc} 0062007 \mathrm{f} 0 \mathrm{fbf}\end{array}$ & $03 / 2011$ & Root exploit \\
\hline Geinimi & com.sgg.spp & $\begin{array}{l}1317 d 996682 \text { f } 4 \text { ae } 4 c c e 6 \\
0 d 90 c 43 f e 3 e 674 f 60 c 22\end{array}$ & $10 / 2011$ & $\begin{array}{l}\text { Information exfiltration; bot- } \\
\text { like capabilities }\end{array}$ \\
\hline Fakeplayer & $\begin{array}{l}\text { org.me.androidappli- } \\
\text { cation1 }\end{array}$ & $\begin{array}{l}\text { 1e993b0632d5bc6f0741 } \\
0 \text { ee31e41dd316435d997 }\end{array}$ & 08/2010 & SMS trojan \\
\hline Bgserv & $\begin{array}{l}\text { com.android.vending. } \\
\text { sectool.v1 }\end{array}$ & $\begin{array}{l}\text { bc2dedad0507a916604f } \\
86167 a 9 f a 306939 e 2080\end{array}$ & $03 / 2011$ & $\begin{array}{l}\text { Information exfiltration; bot- } \\
\text { like capabilities; SMS trojan }\end{array}$ \\
\hline BaseBridge & com.keji.unclear & $\begin{array}{l}508353 \mathrm{~d} 18 \mathrm{cb} 9 f 5544 \mathrm{ble} \\
\mathrm{d} 1 \mathrm{cf} 7 \mathrm{ef} 8 \mathrm{ab} 6 \mathrm{~b} 5552414\end{array}$ & $05 / 2011$ & $\begin{array}{l}\text { Root exploit; SMS trojan } \\
\text { packed as payload }\end{array}$ \\
\hline Plankton & $\begin{array}{l}\text { com.crazyapps.angry. } \\
\text { birds.rio.unlocker }\end{array}$ & $\begin{array}{l}\text { bee } 2661 \text { a } 4 \text { e } 4 b 347 b 5 c d 2 \\
\text { a } 58 f 7 c 4 b 17 b c c 3 e f d 550\end{array}$ & $06 / 2011$ & Dynamic code loading \\
\hline
\end{tabular}

server; we removed the functionality of contacting the remote server to confirm that the malicious functionality works as intended.

\section{THE DATASET}

This section describes the anti-malware products and the malware samples we used for our study. We evaluated ten antimalware tools, which are listed in Table I. There are dozens of free and paid anti-malware offerings for Android from various well-established anti-malware vendors as well as not-so-wellknown developers. We selected the most popular products; in addition, we included Kaspersky and Trend Micro, which were then not very popular but are well established vendors in the security industry. We had to omit a couple of products in the most popular list because they would fail to identify many original, unmodified malware samples we tested. One of the tools, Dr. Web, actually claims that its detection algorithms are resilient to malware modifications.

Our malware set is summarized in Table II. We used a few criteria for choosing malware samples. First, all the anti-malware tools being evaluated should detect the original samples. We here have a question of completeness of the signature set, which is an important evaluation metric for antivirus applications. In this work however, we do not focus on this question. Based on this criterion, we rejected Tapsnake, jSMSHider and a variant of Plankton. Second, the malware samples should be sufficiently old so that signatures against them are well stabilized. All the samples in our set were discovered in or before October 2011. All the samples are publicly available on Contagio Minidump [11].

Our malware set spans over multiple malware kinds. DroidDream [12] and BaseBridge [13] are malware with root exploits packed into benign applications. DroidDream tries to get root privileges using two different root exploits, rage against the cage, and exploid exploit. BaseBridge includes only one exploit, rage against the cage. If these exploits are successful, both DroidDream and BaseBridge install payload applications. Geinimi [14] is a trojan packed into benign applications. It communicates with remote $\mathrm{C} \& \mathrm{C}$ servers and exfiltrates user information. Fakeplayer [15], the first known malware on Android, sends SMS messages to premium numbers, thus costing money to the user. Bgserv [16] is a malware injected into Google's security tool to clean out DroidDream and distributed in third party application markets. It opens a backdoor on the device and exfiltrates user information. Plankton [17] is a malware family that loads classes from additional downloaded dex files to extend its capabilities dynamically.

\section{RESULTS}

As has already been discussed, we transform malware samples using various techniques discussed in Section III and pass them through anti-malware tools we evaluate. We will now briefly describe our methodology and then discuss the findings of our study.

We describe our methodology through Figure 1 and through Tables IV and V, which depict the series of transformations applied to DroidDream and Fakeplayer samples and the detection results on various anti-malware tools. Empty cells in the tables indicate positive detection while cells with ' $x$ ' indicate that the corresponding anti-malware tool failed to detect the malware sample after the given transformations were applied to the sample. The tables reflect a general approach of our study. We begin testing with trivial transformations and then proceed with transformations that are more complex. Each transformation is applied to a malware sample (of course, 


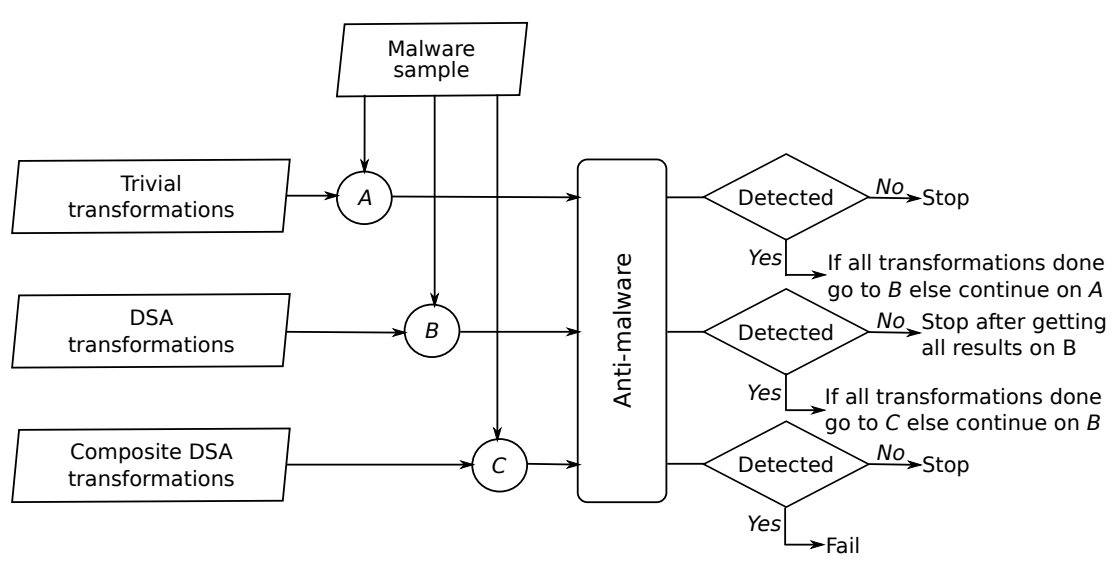

Figure 1: Evaluating anti-malware
TABLE III: Key to Tables IV, V and VI. Transformations coded with single letters are trivial transformations. All others are DSA. We did not need NSA transformations to thwart anti-malware tools.

\begin{tabular}{ll}
\hline Code & Technique \\
\hline P & Repack \\
A & Dissassemble \& assemble \\
RP & Rename package \\
EE & Encrypt native exploit or payload \\
RI & Rename identifiers \\
RF & Rename files \\
ED & Encode strings and array data \\
CR & Reorder code \\
CI & Call indirection \\
JN & Insert junk code \\
\hline All transformations contain P \\
All transformations except P contain A \\
\hline
\end{tabular}

some like exploit encryption apply only in certain cases) and the transformed sample is passed through anti-malware. If detection breaks with trivial transformations, we stop. ${ }^{2}$ Next, we apply all the DSA transformations. If detection still does not break, we apply combinations of DSA transformations. In general there is no well-defined order in which transformations should be applied (in some cases a heuristic works; for example, malware that include native exploits are likely to be detected based on those exploits). Fortunately, in our study, we did not need to apply combinations of more than two transformations to break detection. When applying combinations of transformations, we stopped when detection broke. We do not show the redundant combinations in the tables for the sake of conciseness. The last rows do not form part of our methodology; we construct them manually to show the set of transformations with which all anti-malware tools yield.

Our results with all the malware samples are summarized in Table VI. This table gives the minimal transformations necessary to evade detection for malware-anti-malware pairs. For example, DroidDream requires both exploit encryption and call indirection to evade Dr. Web's detection. These minimal transformations also give insight into what kind of detection signatures are being used. Our tool produces actual malware; we take special precaution to avoid spreading these samples and are careful with whom we share these samples. We next describe our key findings in the light of the detection results. These findings are not meant to be statistical conclusions; yet they give a general idea of the capabilities of anti-malware tools.

Finding 1 All the studied anti-malware products are vulnerable to common transformations. All the transformations appearing in Table VI are easy to develop and apply, redefine only certain syntactic properties of the malware, and are common ways to transform malware. Transformations like identifier renaming and data encryption are easily available

\footnotetext{
${ }^{2}$ All DSA and NSA transformations also result in trivial transformations because of involving disassembling, assembling and repacking. Hence, there is no use in proceeding further.
}

using free and commercial tools [7], [18]. Exploit and payload encryption is also easy to achieve. Although most of current Android malware uses simple techniques, without the use of sophisticated transformations, we point out that some of these transformations may already be seen in the wild in current malware. For example, Geinimi variants have encrypted strings [19]. Similarly, the DroidKungFu malware uses encrypted exploit code [20]; a similar transformation to DroidDream allows easy evasion across almost all the antimalware tools we studied. Finally, there are reports of similar server-side polymorphism as well [6]. In future, it is likely that more and more malware will adopt sophisticated techniques for polymorphism. No transformations just discussed thwart static analysis.

We found that only Dr. Web uses a somewhat more sophisticated algorithm for detection. Our findings indicate that the general detection scheme of Dr. Web is as follows. The set of method calls from every method is obtained. These sets are then used as signatures and the detection phase consists of matching these sets against sets obtained from the sample under test. We also tested Dr. Web against reflection transformation (not shown in the tables) and were able to evade it. This offers another confirmation that signatures are based on method calls. Furthermore, we also found (by limiting our transformations) that only framework API calls matter; calls within the application make no difference. It seems that the matching is somewhat fuzzy (requiring only a threshold percentage of matches) because we found on DroidDream and Fakeplayer that results are positive even when a few classes are removed from the dex file. For these two families, we could create multiple minimal sets of classes that would result in positive detection. As mentioned earlier, Dr. Web indeed claims it has signatures that are resilient to malware modifications. It is difficult to say if the polymorphic resistance of these signatures is any stronger than other signatures depending on identifier names and string and data values. In particular, such signatures do not capture semantic properties of malware such as data and control flow. Our results aptly demonstrate the low resistance. 
TABLE IV: DroidDream transformations and anti-malware failure. Please see Table III for key. ' $x$ ' indicates failure in detection.

\begin{tabular}{|c|c|c|c|c|c|c|c|c|c|c|}
\hline & AVG & Symantec & Lookout & ESET & Dr. Web & Kaspersky & Trend $\mathrm{M}$. & ESTSoft & Zoner & Webroot \\
\hline \multicolumn{11}{|l|}{$\mathrm{P}$} \\
\hline A & & & & & & & & & $\mathrm{x}$ & \\
\hline $\mathrm{RP}$ & $\mathrm{x}$ & & & & & & & $\mathrm{x}$ & $\mathrm{x}$ & \\
\hline $\mathrm{EE}$ & & & $\mathrm{x}$ & & & & & & $\mathrm{x}$ & \\
\hline RI & & $\mathrm{x}$ & & & & & & & $\mathrm{x}$ & $\mathrm{x}$ \\
\hline ED & & & & & & & & & $\mathrm{x}$ & \\
\hline CR & & & & & & & & & $\mathrm{x}$ & \\
\hline $\mathrm{CI}$ & & & & & & & & & $\mathrm{x}$ & \\
\hline $\mathrm{JN}$ & & & & & & & & & $\mathrm{x}$ & \\
\hline $\mathrm{RI}+\mathrm{EE}$ & & $\mathrm{x}$ & $\mathrm{x}$ & $\mathrm{x}$ & & & & & $\mathrm{x}$ & $\mathrm{x}$ \\
\hline $\mathrm{EE}+\mathrm{ED}$ & & & $\mathrm{x}$ & & & $\mathrm{x}$ & & & $\mathrm{x}$ & \\
\hline $\mathrm{EE}+\mathrm{RF}$ & & & $\mathrm{x}$ & & & & $\mathrm{x}$ & & $\mathrm{x}$ & \\
\hline $\mathrm{EE}+\mathrm{CI}$ & & & $\mathrm{x}$ & & $\mathrm{x}$ & & & & $\mathrm{x}$ & \\
\hline $\mathrm{RP}+\mathrm{RI}+\mathrm{EE}+\mathrm{ED}+\mathrm{RF}+\mathrm{CI}$ & $\mathrm{x}$ & $\mathrm{x}$ & $\mathrm{x}$ & $\mathrm{x}$ & $\mathrm{x}$ & $\mathrm{x}$ & $\mathrm{x}$ & $\mathrm{x}$ & $\mathrm{x}$ & $\mathrm{x}$ \\
\hline
\end{tabular}

TABLE V: Fakeplayer transformations and anti-malware failure. Please see Table III for key. ' $\mathrm{x}$ ' indicates failure in detection. EE transformation does not apply for lack of native exploit or payload in Fakeplayer.

\begin{tabular}{|c|c|c|c|c|c|c|c|c|c|c|}
\hline & AVG & Symantec & Lookout & ESET & Dr. Web & Kaspersky & Trend Micro & ESTSoft & Zoner & Webroot \\
\hline \multicolumn{11}{|c|}{ 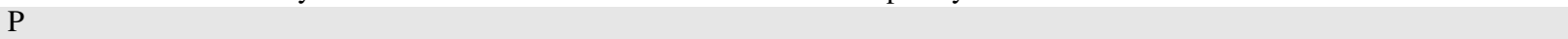 } \\
\hline A & & & & & & & $\mathrm{x}$ & & $\mathrm{x}$ & \\
\hline $\mathrm{RP}$ & & & & & & & $\mathrm{x}$ & $\mathrm{x}$ & $\mathrm{x}$ & $\mathrm{x}$ \\
\hline RI & & & & $\mathrm{x}$ & & $\mathrm{x}$ & $\mathrm{x}$ & & $\mathrm{x}$ & \\
\hline ED & & & & & & & $\mathrm{x}$ & & $\mathrm{x}$ & \\
\hline CR & & & & & & & $\mathrm{x}$ & & $\mathrm{x}$ & \\
\hline CI & & & & & $\mathrm{x}$ & & $\mathrm{x}$ & & $\mathrm{x}$ & \\
\hline JN & & & & & & & $\mathrm{x}$ & & $\mathrm{x}$ & \\
\hline $\mathrm{RP}+\mathrm{RI}$ & $\mathrm{x}$ & $\mathrm{x}$ & $\mathrm{x}$ & $\mathrm{x}$ & & $\mathrm{x}$ & $\mathrm{x}$ & $\mathrm{x}$ & $\mathrm{x}$ & $\mathrm{x}$ \\
\hline $\mathrm{RP}+\mathrm{RI}+\mathrm{CI}$ & $\mathrm{x}$ & $\mathrm{x}$ & $\mathrm{x}$ & $\mathrm{x}$ & $\mathrm{x}$ & $\mathrm{x}$ & $\mathrm{x}$ & $\mathrm{x}$ & $\mathrm{x}$ & $\mathrm{x}$ \\
\hline
\end{tabular}

TABLE VI: Evaluation summary. Please see Table III for key. '+' indicates the composition of two transformations.

\begin{tabular}{lcccccc}
\hline & DroidDream & Geinimi & Fakeplayer & Bgserv & BaseBridge & Plankton \\
AVG & RP & RI & RP + RI & RI & RI & RP + RI \\
Symantec & RI & RI & RP + RI & RI + ED & ED & P \\
Lookout & EE & RI + ED & RP + RI & RI + ED & EE + ED & RI \\
ESET & RI + EE & ED & RI & RI & EE + ED & RI + ED \\
Dr. Web & EE + CI & CI & CI & CI & EE + CI & CI \\
Kaspersky & EE + ED & RI & RI & RI + ED & EE + ED & A \\
Trend M. & EE + RF & RI & A & A & EE + RF & A \\
ESTSoft & RP & RP & RP & RP & RP & RP \\
Zoner & A & RI & A & A & A & RI \\
Webroot & RI & RI & RP & RI & RP & RI \\
\hline
\end{tabular}

Finding 2 At least 43\% signatures are not based on codelevel artifacts. That is, these are based on file names, checksums (or binary sequences) or information easily obtained by the PackageManager API. We also found all AVG signatures to be derived from the content of AndroidManifest only (and hence that of the PackageManager API). In case of AVG, the signatures are based on application component classes or package names or both. Furthermore, this information is derived from AndroidManifest only. We confirmed this by placing a fake AndroidManifest in malware packages and assembling them with the rest of the package kept as it is. This AndroidManifest did not have any of the components or package names declared by the malware applications. The detection was negative for all the malware samples.

Finding $390 \%$ of signatures do not require static analysis of bytecode. Only one of ten anti-malware tools was found to be using static analysis. Names of classes, methods, and fields, and all the strings and array data are stored in the classes. dex file as they are and hence can be obtained by content matching. The only signatures requiring static analysis of bytecode are those of Dr. Web because it extracts API calls made in various methods.

Finding 4 Anti-malware tools have evolved towards content-based signatures over the past one year. We studied compare our findings that we obtained in February 2012 (Table VII) to our present findings obtained in February 2013 (Table VI). Some of the anti-malware tools have changed considerably for the same malware samples. Last year, $45 \%$ of the signatures were evaded by trivial transformations, i.e., repacking and assembling/disassembling. Such signatures have virtually no resilience against polymorphism. Our present results show a marked decrease in this fraction to $16 \%$. We find that in all such cases where we see changes, antimalware authors have moved to content-based matching, such 
which signatures can be developed. The use of reflection simply hides away the edges in the call graph. If the method names used for reflective invocations are encrypted, these edges are rendered completely opaque to static analysis. Furthermore, it is possible to use function outlining to thwart any forms of intra-procedural analysis as well. Owing to these limitations, the use of dynamic monitoring is essential.

Recall that anti-malware tools in Android are unprivileged third party applications. This impedes many different kinds of dynamic monitoring that may enhance malware detection. We believe special platform support for anti-malware applications is essential to detect malware amongst stock Android applications. This can help malware detection in several ways. For example, a common way to break evasion by code encryption is to scan the memory at runtime. The Android runtime could provide all the classes loaded using user-defined class loaders to the anti-malware application. The loaded classes are already decrypted and anti-malware tools can analyze them easily.

Google Bouncer performs offline dynamic analysis for malware detection [26]. Such scanning has its own problems, ranging from detection of the dynamic environment to the malicious activity not getting triggered in the limited time for which the emulation runs [27], [28]. We therefore believe offline emulation must be supplemented by strong static analysis or real-time dynamic monitoring.

\section{RELATED WORK}

Evaluating Anti-malware Tools. Zheng et al. [29] also studied the robustness of anti-malware against Android malware recently using a tool called ADAM. ADAM implements only a few transformations, renaming methods, introducing junk methods, code reordering, and string encoding, in addition to repacking and assembling/disassembling. Our set of transformations is much more comprehensive and includes renaming packages, classes, encoding array data, inserting junk statements, encrypting payloads and native exploits, reflection, and bytecode encryption as well. Finally, we also have composite transformations. Many of the additional transformations, including the composite ones, were crucial for evading antimalware tools. Based on the above, we point out that ADAM is not always able to evade an anti-malware tool. Rather than attempting complete evasion, it simply offers percentages depicting how many variants were detected by the anti-malware tools (and these percentages are also very high). In contrast, our framework is comprehensive, aimed towards complete evasion of all anti-malware tools. We believe our results make a clear statement - all anti-malware tools can be evaded using common obfuscation techniques. Unlike ADAM, our result is able to highlight the severity of the problem and is easily accessible.

Christodorescu and Jha [5] conducted a study similar to ours on desktop anti-malware applications nine years ago. They also arrived at the conclusion that these applications have low resilience against malware obfuscation. Our study is based on Android anti-malware, and we include several aspects in our study that are unique to Android. Furthermore, our study dates after many research works (see below) on obfuscation resilient detection, and we would expect the proposed techniques to be readily integrated into new commercial products.

Obfuscation Techniques. Collberg et al. [30] review and propose different types of obfuscations. DroidChameleon provides only a few of the transformations proposed by them. Nonetheless, the set of transformations provided in DroidChameleon is comprehensive in the sense that they can break typical static detection techniques used by anti-malware. Off-the-shelf tools like Proguard [7] and Klassmaster [18] provide renaming of classes and class members, flow obfuscation, and string encryption. While the goal of these tools is to evade manual reverse engineering, we aim at thwarting analysis by automatic tools.

Obfuscated Malware Detection. Obfuscation resilient detection is based on semantics rather than syntac. As discussed earlier, Christodorescu et al. [21] present one such technique. Christodorescu et al. [31] and Fredrikson et al. [22] attempt to generate semantics based signatures by mining malicious behavior automatically. Kolbitsch et al. [23] also propose similar techniques. The last three works are for behaviorbased detection and use different behavior representations such as data dependence graphs and information flows between system calls. Due to lower privileges for anti-malware tools on Android, these approaches cannot directly apply to these tools presently. Sequence alignment from bioinformatics [32], [33] has also been applied to malware detection and related problems [34], [35]. Further work is also there to compute statistical significance of scores given by these classical sequence alignment algorithms [36], [37]. It may be possible to adapt such techniques to detect transformed malware with high performance.

Smartphone Malware Research. Many works have been done towards discovery and characterization of smartphone malware [38], [39], [40], [41], [42], [43], [44]. Our work is distinct from these as we try to evaluate the efficacy of existing tools against transformed malware.

\section{CONCLUSION}

We evaluated ten anti-malware products on Android for their resilience against malware transformations. To facilitate this, we developed DroidChameleon, a systematic framework with various transformation techniques. Our findings using transformations of six malware samples show that all the anti-malware products evaluated are susceptible to common evasion techniques and may succumb to even trivial transformations not involving code-level changes. Finally, we explored possible ways in which the current situation may be improved and next-generation solutions may be developed. As future work, we plan to perform a more comprehensive evaluation using a much larger number of malware samples and anti-malware tools. Interested readers are referred to http://list.cs.northwestern.edu/mobile for related source code and technical reports.

\section{REFERENCES}

[1] V. Rastogi, Y. Chen, and X. Jiang, "DroidChameleon: Evaluating Android anti-malware against transformation attacks," in Proceedings of ACM ASIACCS 2013, May 2013. 
[2] CNET, February 2013, http://news.cnet.com/8301-1035_3-5756940294/android-ios-combine-for-91-percent-of-market/.

[3] McAfee, "Mcafee threats report: Third quarter 2011," http://www. mcafee.com/us/resources/reports/rp-quarterly-threat-q3-2011.pdf.

[4] F-Secure, "Mobile threat report Q3 2012," http://www.fsecure.com/static/doc/labs_global/Research/Mobile\%20Threat\% 20Report\%20Q3\%202012.pdf.

[5] M. Christodorescu and S. Jha, "Testing malware detectors," in Proceedings of the 2004 ACM SIGSOFT International Symposium on Software Testing and Analysis, ser. ISSTA '04. ACM, 2004.

[6] Symantec, "Server-side polymorphic android applications," http://www.symantec.com/connect/blogs/server-side-polymorphicandroid-applications.

[7] "ProGuard," http://proguard.sourceforge.net/.

[8] R. Komondoor and S. Horwitz, "Semantics-preserving procedure extraction," in In POPL. ACM Press, 2000, pp. 155-169.

[9] "Smali: An assembler/disassembler for Android's dex format," http:// code.google.com $/ \mathrm{p} / \mathrm{smali} /$.

[10] "Android-apktool: A tool for reengineering Android apk files," http: //code.google.com/p/android-apktool/.

[11] M. Parkour, "Contagio Mobile. Mobile malware mini dump," http: //contagiominidump.blogspot.com/.

[12] Lookout, "Update: Security alert: DroidDream malware found in official Android Market," http://blog.mylookout.com/blog/2011/03/01/securityalert-malware-found-in-official-android-market-droiddream/.

[13] "Android.Basebridge - Symantec," http://www.symantec.com/ security_response/writeup.jsp?docid=2011-060915-4938-99.

[14] "Android.Geinimi - Symantec," http://www.symantec.com/security_ response/writeup.jsp?docid=2011-010111-5403-99.

[15] "AndroidOS.FakePlayer - Symantec," http://www.symantec.com/ security_response/writeup.jsp?docid=2010-081100-1646-99.

[16] "Android.Bgserv - Symantec," http://www.symantec.com/security response/writeup.jsp?docid=2011-031005-2918-99.

[17] "Plankton," http://www.csc.ncsu.edu/faculty/jiang/Plankton/.

[18] "Zelix Klassmaster," http://www.zelix.com/klassmaster/.

[19] Lookout, "Geinimi trojan technical analysis," http://blog.mylookout. com/blog/2011/01/07/geinimi-trojan-technical-analysis/.

[20] "DroidKungFu," http://www.csc.ncsu.edu/faculty/jiang/DroidKungFu. html.

[21] M. Christodorescu, S. Jha, S. Seshia, D. Song, and R. Bryant, "Semantics-aware malware detection," in Security and Privacy, 2005 IEEE Symposium on. IEEE, 2005, pp. 32-46.

[22] M. Fredrikson, S. Jha, M. Christodorescu, R. Sailer, and X. Yan, "Synthesizing near-optimal malware specifications from suspicious behaviors," in Security and Privacy (SP), 2010 IEEE Symposium on. IEEE, 2010, pp. 45-60.

[23] C. Kolbitsch, P. Comparetti, C. Kruegel, E. Kirda, X. Zhou, and $\mathrm{X}$. Wang, "Effective and efficient malware detection at the end host," in Proceedings of the 18th Conference on USENIX Security Symposium. USENIX Association, 2009, pp. 351-366.

[24] P. Saxena, R. Sekar, and V. Puranik, "Efficient fine-grained binary instrumentationwith applications to taint-tracking," in Proceedings of the 6th annual IEEE/ACM international symposium on Code generation and optimization. ACM, 2008, pp. 74-83.

[25] L. Harris and B. Miller, "Practical analysis of stripped binary code," ACM SIGARCH Computer Architecture News, vol. 33, no. 5, pp. 6368, 2005.

[26] H. Lockheimer, "Android and security," February 2012, http:// googlemobile.blogspot.com/2012/02/android-and-security.html.

[27] J. Oberheide, "Dissecting android's bouncer," June 2012, https://blog. duosecurity.com/2012/06/dissecting-androids-bouncer/.

[28] R. Whitwam, "Circumventing Google's Bouncer, Android's antimalware system," June 2012, http://www.extremetech.com/computing/ 130424-circumventing-googles-bouncer-androids-anti-malwaresystem.

[29] M. Zheng, P. Lee, and J. Lui, "Adam: An automatic and extensible platform to stress test Android anti-virus systems," DIMVA, July 2012.

[30] C. Collberg, C. Thomborson, and D. Low, "A taxonomy of obfuscating transformations," Department of Computer Science, The University of Auckland, New Zealand, Tech. Rep., 1997.

[31] M. Christodorescu, S. Jha, and C. Kruegel, "Mining specifications of malicious behavior," in Proceedings of the the 6th Joint Meeting of the European Software Engineering Conference and the ACM SIGSOFT Symposium on The Foundations of Software Engineering, ser. ESEC-FSE '07. ACM, 2007.
[32] S. B. Needleman and C. D. Wunsch, "A general method applicable to the search for similarities in the amino acid sequence of two proteins." Journal of Molecular Biology, vol. 48, no. 3, pp. 443-453, March 1970.

[33] T. F. Smith and M. S. Waterman, "Identification of Common Molecular Subsequences." Journal of Molecular Biology, vol. 147, no. 1, pp. 195197, 1981.

[34] X. Jiang and X. Zhu, "vEye: Behavioral footprinting for self-propagating worm detection and profiling," Knowledge and Information Systems, vol. 18, no. 2, pp. 231-262, 2009.

[35] G. Wondracek, P. M. Comparetti, C. Kruegel, E. Kirda, and S. S. S. Anna, "Automatic network protocol analysis," in 15th Symposium on Network and Distributed System Security (NDSS), 2008.

[36] A. Agrawal and X. Huang, "Pairwise statistical significance of local sequence alignment using multiple parameter sets and empirical justification of parameter set change penalty," BMC Bioinformatics, vol. 10, no. Suppl 3, p. S1, 2009.

[37] — - "Pairwise statistical significance of local sequence alignment using sequence-specific and position-specific substitution matrices," IEEE/ACM Transactions on Computational Biology and Bioinformatics (TCBB), vol. 8, no. 1, pp. 194-205, 2011.

[38] Y. Zhou, Z. Wang, W. Zhou, and X. Jiang, "Hey, you, get off of my market: Detecting malicious apps in official and alternative Android markets," in Proceedings of the 19th Network and Distributed System Security Symposium, ser. NDSS '12, 2012.

[39] M. Grace, Y. Zhou, Q. Zhang, S. Zou, and X. Jiang, "Riskranker: scalable and accurate zero-day android malware detection," in Proceedings of the 10th International Conference on Mobile Systems, Applications, and Services, ser. MobiSys '12. ACM, 2012.

[40] H. Peng, C. Gates, B. Sarma, N. Li, Y. Qi, R. Potharaju, C. Nita-Rotaru, and I. Molloy, "Using probabilistic generative models for ranking risks of android apps," in Proceedings of the 2012 ACM conference on Computer and communications security, 2012.

[41] I. Burguera, U. Zurutuza, and S. Nadjm-Tehrani, "Crowdroid: behaviorbased malware detection system for android," in Proceedings of the 1st ACM workshop on Security and privacy in smartphones and mobile devices. ACM, 2011, pp. 15-26.

[42] Y. Zhou and X. Jiang, "Dissecting android malware: Characterization and evolution," Security and Privacy, IEEE Symposium on, 2012.

[43] Y. Nadji, J. Giffin, and P. Traynor, "Automated remote repair for mobile malware," in Proceedings of the 27th Annual Computer Security Applications Conference. ACM, 2011, pp. 413-422.

[44] V. Rastogi, Y. Chen, and W. Enck, "Appsplayground: Automatic security analysis of smartphone applications," in Proceedings of ACM CODASPY 2013, February 2013.

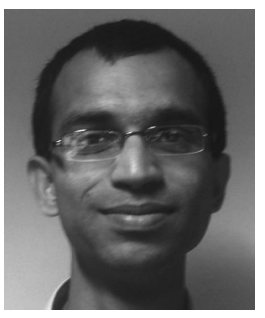

Vaibhav Rastogi is a $\mathrm{PhD}$ candidate in the $\mathrm{De}$ partment of Electrical Engineering and Computer Science at Northwestern Univeristy. His research interests include web security and mobile device security

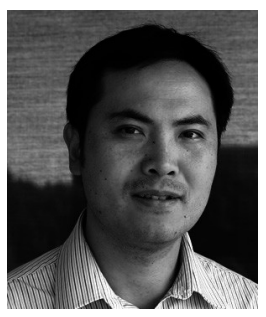

Yan Chen is an Associate Professor in the Department of Electrical Engineering and Computer Science at Northwestern University, Evanston, IL. $\mathrm{He}$ received his Ph.D. in Computer Science from University of California at Berkeley in 2003. His research interests include network security, measurement and diagnosis for large scale networks and distributed systems. Based on Google Scholar, his papers have been cited for over 5,600 times and his $\mathrm{h}$-index is 29 .

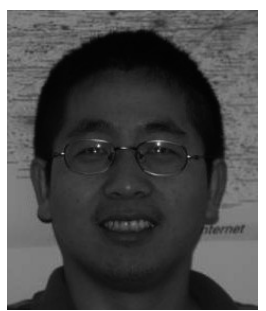

Xuxian Jiang is an Associate Professor in the Computer Science Department and a core member of the Cyber Defense Lab at the North Carolina State University. He received his $\mathrm{PhD}$ degree in Computer Science from Purdue University in 2006. His research interests are mainly in smartphones, hypervisors, and malware defense. 